\title{
Comparative study between local anesthesia and general anesthesia in the treatment of primary spontaneous pneumothorax
}

\author{
Joonho Jung ${ }^{1}$, Do Hyung Kim², Joohyung Son ${ }^{2}$, Sung Kwang Lee ${ }^{2}$, Bong Soo Son ${ }^{2}$ \\ ${ }^{1}$ Department of Thoracic and Cardiovascular Surgery, Ajou University Hospital, Suwon, Korea; ${ }^{2}$ Department of Thoracic and Cardiovascular \\ Surgery, Pusan National University Yangsan Hospital, Medical Research Institute of Pusan National University, Busan, Korea \\ Contributions: (I) Conception and design: J Jung, DH Kim, J Son; (II) Administrative support: All authors; (III) Provision of study materials or \\ patients: J Jung, DH Kim, J Son; (IV) Collection and assembly of data: J Jung, DH Kim, J Son; (V) Data analysis and interpretation: J Jung, DH Kim; \\ (VI) Manuscript writing: All authors; (VII) Final approval of manuscript: All authors. \\ Correspondence to: Do Hyung Kim, MD. Department of Thoracic and Cardiovascular Surgery, Pusan National University Yangsan Hospital, Geumo- \\ ro 20, Beomeo-ri, Mulgeum-eup, Yangsan-si, Gyeongsangnam-do 626-770, Korea. Email: yumccs@nate.com.
}

Background: There is increasing interest in non-intubated video-assisted thoracoscopic surgery (VATS). At present, this method is used in various types of thoracic surgery. Especially, simple wedge resection via VATS is thought to be an optimal indication of non-intubated VATS. This study was performed to evaluate the usefulness of VATS bullectomy under local anesthesia by comparison with bullectomy under general anesthesia.

Methods: A total of 183 cases of wedge resection under general anesthesia and 52 cases of wedge resection under local anesthesia were examined. Medical records were retrospectively reviewed to assess the feasibility, usability, and cost effectiveness of wedge resection under local anesthesia. The preoperative clinical parameters, including age, sex, and the number of pneumothorax episodes and previous operations, were used to match cases and controls.

Results: There were no significant differences between the local and general anesthesia groups in operative time (57.5 \pm 23.1 and $56.6 \pm 25.6 \mathrm{~min}$, respectively; $\mathrm{P}=0.857)$, type of operation [single-incision thoracoscopic surgery (SITS), $\mathrm{n}=48$ and $\mathrm{n}=47$, respectively; multi-port-VATS (M-VATS), $\mathrm{n}=4$ and $\mathrm{n}=5$, respectively; $\mathrm{P}=0.730$ ], or chest tube indwelling time (3.6 \pm 1.5 and $4.4 \pm 2.5$ days, respectively; $\mathrm{P}=0.064)$. The mean times from arrival at the operating room $(\mathrm{OR})$ to skin incision $(16.4 \pm 12.3$ and $46.4 \pm 17.2$ min, respectively; $\mathrm{P}<0.001)$, and from the end of surgery to arrival at the general ward $(36.0 \pm 25.6$ and $58.1 \pm 20.9 \mathrm{~min}$, respectively; $\mathrm{P}<0.001)$ were significantly shorter in the local anesthesia group than the general anesthesia group. The total cost was significantly lower in the local anesthesia group than in the general anesthesia group $(4,890.6 \pm 717.1$ and 5,739.1 $\pm 1,154.6$, respectively; $\mathrm{P}<0.001)$.

Conclusions: Local anesthesia shortened the overall hospital stay by reducing the interval between admission and surgery, allowing immediate ambulation after surgery. In addition, this method reduced costs by avoiding the need for anesthesia.

Keywords: Bullectomy; non-intubated; video-assisted thoracoscopic (VAT); pneumothorax

Submitted Jul 07, 2019. Accepted for publication Sep 10, 2019.

doi: $10.21037 /$ atm.2019.09.89

View this article at: http://dx.doi.org/10.21037/atm.2019.09.89

\section{Introduction}

General anesthesia during surgery is likely to cause more cognitive dysfunction than regional anesthesia because of deep sedation; furthermore, the use of muscle relaxants can cause diaphragm dysfunction and one-lung ventilation may increase the ventilation/perfusion mismatch due to atelectasis. On the other hand, if spontaneous breathing under minimal sedation is maintained during lung surgery, 


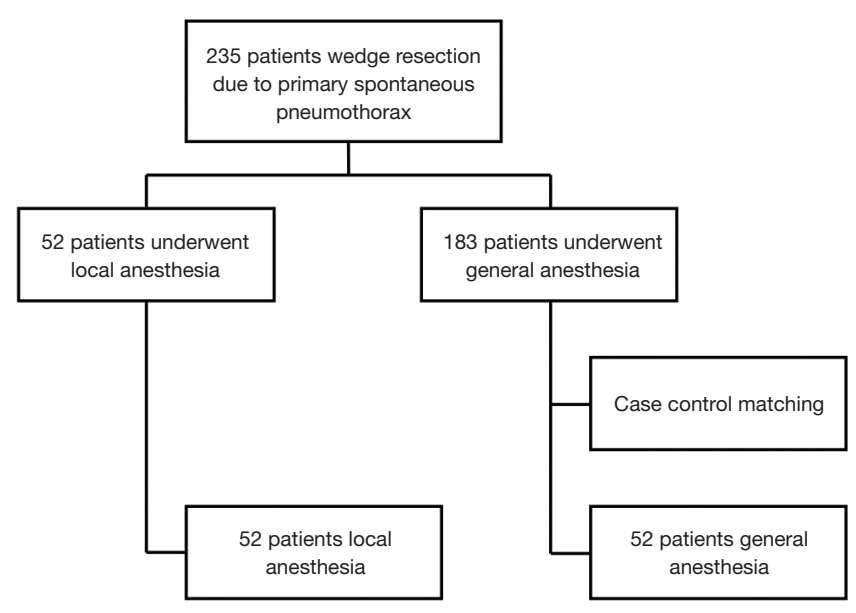

Figure 1 Flowchart of patient's inclusion and matching results. After case control matching, we analyzed 52 patients in each group.

effective breathing can maintain the pressure in alveolar lungs on the surgical side, minimizing the occurrence of ventilation/perfusion mismatch by preventing hypoxiainduced pulmonary vasoconstriction (1-3).

If possible, it is advantageous to perform surgery under spontaneous breathing rather than mechanical ventilation. However, application of non-intubated video-assisted thoracoscopic surgery (VATS) is difficult and a high level of surgical skill is required (4). It is necessary to find appropriate indications and increase the availability of nonintubated VATS, rather than applying this methodology to all types of thoracic surgery. We considered VATS bullectomy in cases of primary spontaneous pneumothorax (PSP) as the most appropriate indication based on the suggestion of (1) the thoracic surgeon who first reported non-intubated VATS. This study was performed to compare the feasibility of VATS bullectomy under local anesthesia with bullectomy under general anesthesia.

\section{Methods}

This study was approved by the Institutional Review Board of Pusan National University Yangsan Hospital (IRB-No. 05-2019-012). We performed single-incision thoracoscopic surgery (SITS) for treatment of PSP between July 2011 and May 2017. We first began to perform non-intubated VATS with local anesthesia in January 2016. A total of 235 patients were identified, with 52 treated under local anesthesia and 140 treated under general anesthesia. The general indications for VATS bullectomy were as follows:
(I) recurrent episodes of spontaneous pneumothorax on the ipsilateral or contralateral side; (II) prolonged air leakage for more than 5 days after insertion of a chest tube; and (III) blebs evident on computed tomography (CT). To ensure fair comparison between the two surgeries, casecontrol matching of non-intubated VATS and conventional VATS was performed using MedCalc software (MedCalc, Mariakerke, Belgium). The included parameters were age, sex, and the number of pneumothorax episodes and previous operations. A one-to-one match was achieved using the nearest neighbor-matching algorithm. A flowchart summarizing patient inclusion and matching results is shown in Figure 1.

We measured operation time, blood loss during surgery, duration of chest tube drainage, volume of fluid drained during the first 24 hours, duration of postoperative hospital stay, postoperative complications, and Visual Analog Scale (VAS) pain scores on postoperative days 0,1 , and 2. All patients were followed up in our outpatient clinic after 1 week, and during months 3 and 6 after discharge. All clinical data were analyzed retrospectively and compared between the two groups.

\section{Surgical procedure and anesthesia}

\section{Single-incision VATS bullectomy under general anesthesia}

Each patient was placed in the lateral decubitus position and intubated with a double-lumen endotracheal tube. After standard skin preparation, a skin incision less than $2 \mathrm{~cm}$ in length was made at the anterior axillary line of the fourth or fifth intercostal space to treat the target lesion in the lung apex. An extra-small wound protector (U-Tractor; Yuwon Meditech, WonJu-si, Kangwondo, South Korea) was inserted. The lesion for resection was identified through the incision using a thoracoscope $5 \mathrm{~mm}$ in diameter angled at $30^{\circ}$, and a 1-0 Prolene suture (Ethicon, Somerville, NJ, USA) was passed from outside the chest wall, above the bleb lesion, to the interior of the chest cavity. The anchoring suture thread was passed through the third intercostal space anterior to or, on the midaxillary line of, the chest wall (the latter route was chosen when the target bleb was in the apex of the upper lobe). Anchoring sutures were placed in front of the bleb, and the needle was pulled out through the incision site. After placing bidirectional traction sutures, the lesion for resection was lifted by pulling the thread on the side of the chest wall to create an angle, which enabled a stapler to be used to excise the lesion. First, stapling 
was performed by inserting the scope and an articulating endostapler (Ethicon, Cincinnati, OH, USA) in parallel. After the first stapling, a second stapler was inserted and directed toward the previously resected surface. The maximal possible resection was achieved by pushing the stapler, because the lesion was pulled to the center of the chest cavity by applying force to the thread on the incisional side. Once the resection was completed, the tissue was removed by pulling on the thread on the incisional side, and a nonwoven polyglycolic acid Neoveil sheet (Gunze, Aybe, Japan) was used together with fibrin glue to reinforce the stapling line. A 20-F chest tube was inserted into the pleural cavity through the incision site, and the wound was closed around the tube.

\section{Single-incision VATS bullectomy under local anesthesia using a high-flow nasal cannula}

After entering the operation room (OR), midazolam $(0.06 \mathrm{mg} / \mathrm{kg})$ was injected intravenously 15 minutes before the operation. During the procedure, patients breathed spontaneously through a high-flow nasal cannula to maintain oxygen saturation $>95 \%$. Each patient's electrocardiogram, heart rate, blood pressure, pulse oxygen saturation $\left(\mathrm{SpO}_{2}\right)$, and respiratory rate were monitored continuously, and the patient was placed in the lateral decubitus position. After standard skin preparation, remifentanil $(0.1-0.3 \mathrm{mg})$ was injected intravenously and a skin incision about $2.5 \mathrm{~cm}$ in length was made at the anterior axillary line of the fourth or fifth intercostal space to treat the target lesion under local anesthesia with $1 \%$ lidocaine. As all instruments must be used within a single incision, in contrast to the bidirectional anchoring suture technique, the average incision size was about $5 \mathrm{~mm}$ larger than in the general anesthesia technique. After intercostal dissection, an extra-small wound protector (U-Tractor; Yuwon Meditech) was inserted. The lesion for resection was identified through the incision using a thoracoscope $5 \mathrm{~mm}$ in diameter angled at $30^{\circ}$ and an endograsper. After confirmation of the lesion for resection, conventional single-incision wedge resection was performed with the endograsper and an articulating endostapler (Ethicon) in parallel, instead of the bidirectional anchoring suture technique. Once resection was completed, the tissue was removed by pulling on the thread on the incisional side, and a nonwoven polyglycolic acid Neoveil sheet (Gunze) was used together with fibrin glue to reinforce the stapling line. A 20-F chest tube was inserted into the pleural cavity through the incision site, and the wound was closed around the tube.

\section{Statistical analyses}

Qualitative variables are reported as numbers and percentages, and quantitative variables are reported as means \pm standard deviation (SD). Data analysis was performed using IBM SPSS Statistics (ver. 22.0; IBM Corp., Armonk, NY, USA). Variables were compared using Student's $t$-test, the $\chi^{2}$ test, and the Mann-Whitney $\mathrm{U}$ test. A two-sided $\mathrm{P}<0.05$ was taken to indicate statistical significance.

\section{Results}

\section{Demographics and patient characteristics}

Table 1 compares the demographics and characteristics of patients undergoing VATS under local anesthesia and general anesthesia. Before case-control matching, the local anesthesia $(\mathrm{n}=52)$ and general anesthesia $(\mathrm{n}=183)$ showed no significant differences in age $(20.4 \pm 7.0$ and $22.9 \pm 9.2$ years, respectively; $\mathrm{P}=0.061$ ), number of pneumothorax episodes (one, $n=51$ and $n=166$; two, $n=1$ and $n=15$; three or more, $\mathrm{n}=0$ and $\mathrm{n}=2$, respectively; $\mathrm{P}=0.081$ ), operation site (right, $\mathrm{n}=22$ and $\mathrm{n}=101$; left, $\mathrm{n}=28$ and $\mathrm{n}=80$; both, $\mathrm{n}=2$ and $\mathrm{n}=2$, respectively; $\mathrm{P}=0.062)$, or previous operation history $(\mathrm{n}=5$ and $\mathrm{n}=28$, respectively; $\mathrm{P}=0.156$ ). However, there was a significant difference in sex ratio between the two groups $(\mathrm{P}=0.024)$.

We also performed case-control matching in the general anesthesia group to allow appropriate comparison of the two groups, and the results are shown in Table 1. After casecontrol matching, the number of patients in the general anesthesia group was 52, and compared with the local anesthesia group, there was no significant difference in age (20.4 \pm 7.0 and 19.3 \pm 4.6 , respectively; $\mathrm{P}=0.346)$, number of pneumothorax episodes (one, $\mathrm{n}=51$ and $\mathrm{n}=51$; two, $\mathrm{n}=1$ and $\mathrm{n}=1$; three or more, $\mathrm{n}=0$ and $\mathrm{n}=0$, respectively; $\mathrm{P}=1.000$ ), operation site (right, $n=22$ and $n=23$; left, $n=28$ and $n=29$; both, $\mathrm{n}=2$ and $\mathrm{n}=0$, respectively; $\mathrm{P}=0.583$ ), or previous operation history ( $n=47$ and $n=47$, respectively; $P=1.000)$. There was also no difference in sex ratio between the two groups after propensity score matching (50:2 and 50:2, respectively; $\mathrm{P}=1.000$ ). Therefore, similarity was increased in both groups and there were no significant differences in demographics or patient characteristics. The clinical outcomes and perioperative results were compared between the two groups. There was no case that convert to general anesthesia and intubation during operation under local anesthesia. 
Table 1 Demographics and clinical patient characteristics

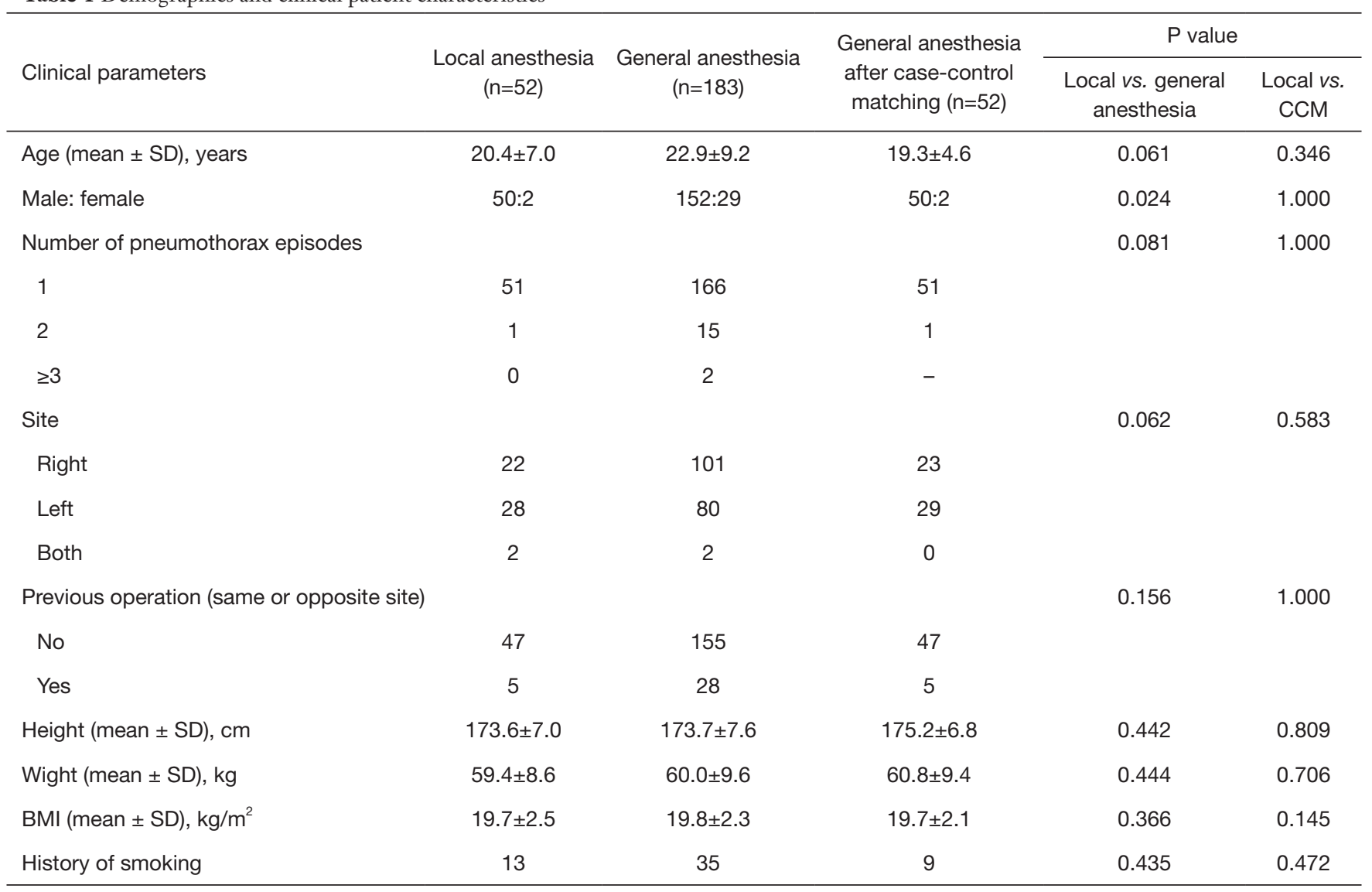

CCM, case-control matching; BMI, Body mass index.

\section{Perioperative results after case-control matching}

Table 2 shows the perioperative results according to the type of operation, operation time, hospital stay, and cost of operation for the two groups after case-control matching. There was no case that convert to general anesthesia and intubation during operation under local anesthesia. There was no significant difference in the type of operation between the local and general anesthesia groups [SITS, $\mathrm{n}=48$ and $\mathrm{n}=47$; multi-port-VATS (M-VATS), $\mathrm{n}=4$ and $\mathrm{n}=5$, respectively; $\mathrm{P}=0.730]$. Local anesthesia allows easy access to the OR. In the local anesthesia group, the time from admission to operation was $1.3 \pm 1.4$ days (general anesthesia group, $4.1 \pm 2.7$ days; $\mathrm{P}<0.001)$. The local anesthesia group showed significantly reduced time from arrival at the OR to skin incision compared to the general anesthesia group $(16.4 \pm 12.3$ and $46.4 \pm 17.2 \mathrm{~min}$, respectively; $\mathrm{P}<0.001)$. In addition, time from end of surgery to arrival at the general ward was significantly shorter in the local anesthesia group compared to the general anesthesia group $(36.0 \pm 25.6$ and $58.1 \pm 20.9$ min, respectively; $\mathrm{P}<0.001)$. Similarly, hospital stay was significantly shorter in the local anesthesia group than the general anesthesia group $(5.4 \pm 2.3$ and $9.2 \pm 4.7$ days, respectively; $\mathrm{P}<0.001$ ).

The amount of bleeding was also significantly lower in the local anesthesia group compared to the general anesthesia group $(7.0 \pm 7.1$ and $22.1 \pm 24.4 \mathrm{~mL}$, respectively; $\mathrm{P}<0.001)$. As there was no difference in operative methods between the local and general anesthesia groups, there was no significant difference in operation time $(57.5 \pm 23.1$ and 56.6 $\pm 25.6 \mathrm{~min} ; \mathrm{P}=0.857)$. Chest tube indwelling time and postoperative hospital stay were compared. The duration of chest tube placement was $3.6 \pm 1.5$ days in the local anesthesia group and $4.4 \pm 2.5$ days in the general anesthesia group ( $\mathrm{P}=0.064)$. The time from operation to discharge was $4.0 \pm 1.9$ days in the local anesthesia group and 5.0 \pm 3.6 days in the general anesthesia group $(\mathrm{P}=0.067)$. Although both chest tube indwelling time and time from 
Table 2 Comparison of clinical results between local anesthesia and general anesthesia

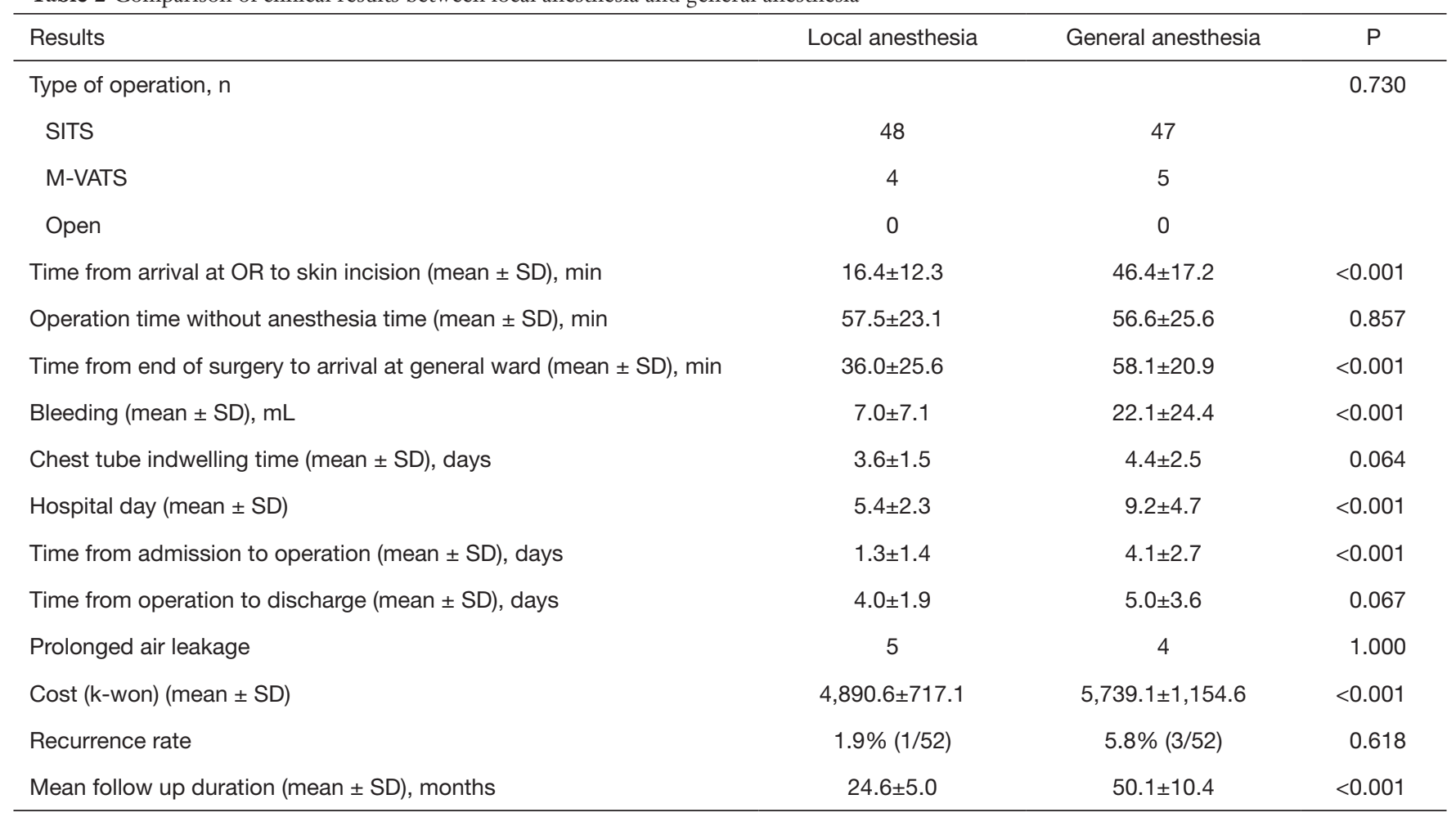

SITS, single-incision thoracoscopic surgery; M-VATS, multi-port video-assisted thoracoscopic surgery; OR, operating room.

operation to discharge were 1 day shorter in the local anesthesia group, the differences were not significant. The common but potentially severe complications after thoracic surgery, such as bleeding, wound infection and pneumonia, were not occurred in both groups. But prolonged air leakage, defined as air leakage presents 5 or more days after operation, was noted in 5 cases in local anesthesia group, 4 in general anesthesia group $(\mathrm{P}=1.000)$. The total cost of operation was significantly lower in the local anesthesia group than the general anesthesia group $(4,890.6 \pm 717.1$ and $5,739.1 \pm 1,154.6$, respectively; $\mathrm{P}<0.001)$.

Pain scores immediately after surgery, on postoperative days 1 and 2, 1 day before and after removal of chest tube were compared between the two groups (Figure 2). There was no significant difference in pain score immediately after the operation between the local and general anesthesia groups $\mathrm{P}=0.311$ ), but the local anesthesia group showed significantly lower pain scores on the first and second days postoperatively $\mathrm{P}=0.005$ and $\mathrm{P}=0.036$, respectively). There were no significant differences in pain scores 1 day before removal of chest tube or 1 day after removal of the chest tube $\mathrm{P}=0.058$ and $\mathrm{P}=0.388$, respectively).
The recurrence rate after bullectomy was lower in the local anesthesia group $(1.9 \%, 1 / 52)$ than the general anesthesia group $(5.8 \%, 3 / 52)$, but there was no statistical significance $(\mathrm{P}=0.618)$. The mean duration of follow up was shorter in the local anesthesia group $(\mathrm{P}<0.001)$.

\section{Discussion}

Previous reports on non-intubated VATS have mostly discussed anatomical resection of lung cancer, but there have also been reports in the minor surgery field (5-7). Pompeo et al. (8) introduced non-intubated VATS in various minor thoracic surgery areas, including pleural effusion, pulmonary biopsies, mediastinal biopsies, metastatic tumors, spontaneous pneumothorax, empyema thoracis, and emphysematous bulla VATS, and reported its superiority to general anesthesia. However, the surgeon who first reported non-intubated VATS (1) suggested that there are limitations to the applicability of this method to minor surgery in all regions. It is necessary to select an appropriate initial indication for establishment of the program. It is reasonable to increase indications from simple lung resection, which at 


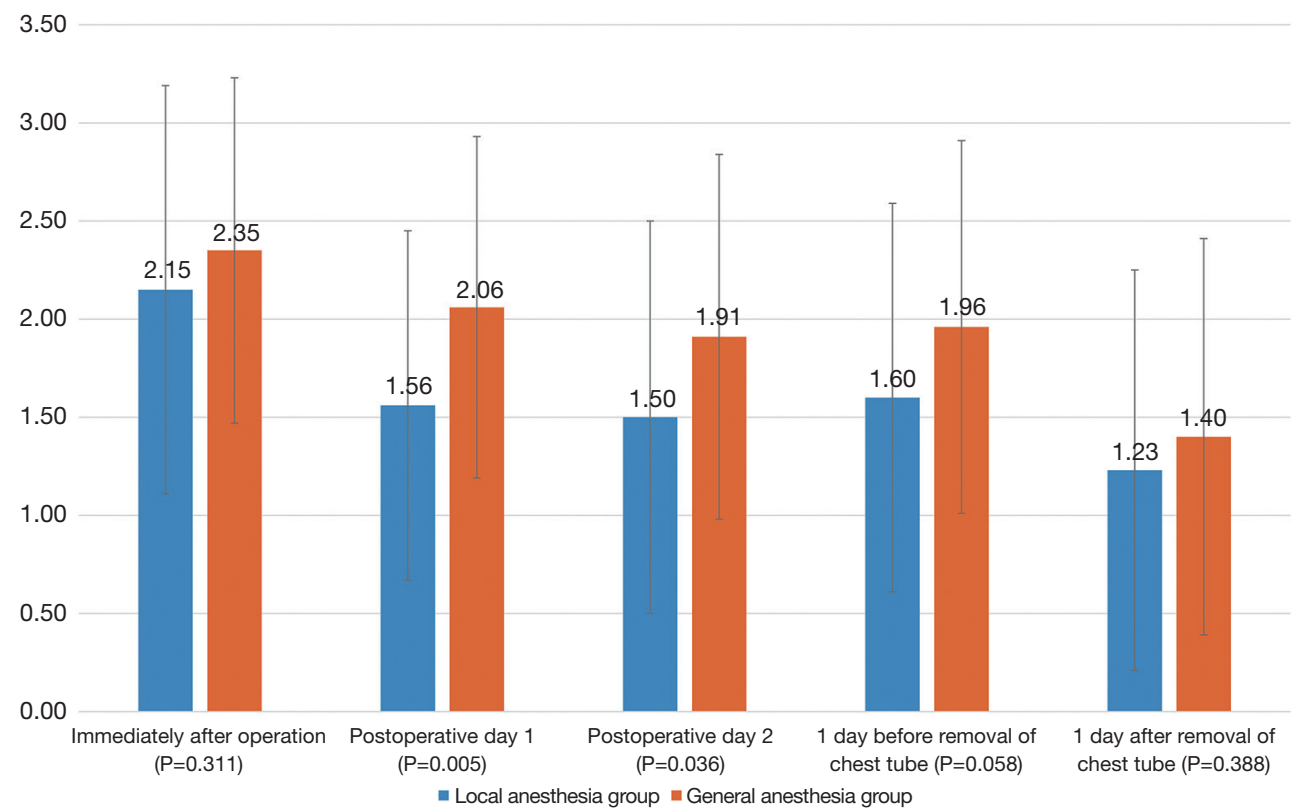

Figure 2 Comparison of visual analog pain scores between local anesthesia and general anesthesia. The pain score was lower in the local anesthesia group in postoperative day 1 \& 2 than the general anesthesia group.

present is the most appropriate indication to replace general anesthesia (9).

We first introduced non-intubated VATS as the most ideal indication in PSP patients. PSP usually occurs in young patients who have good pulmonary function, are hemodynamically stable, and have limited comorbidities for surgery and can better tolerate a moderate degree of hypercapnia during surgery. Bullectomy was considered the first surgery for surgeons with little clinical experience of non-intubated VATS. However, there have been few reports on the use of non-intubated VATS in spontaneous pneumothorax, and additional clinical data are required. Bullectomy was first reported in PSP pneumothorax patients in 2007 (10). Despite the relative paucity of clinical data, non-intubated VATS bullectomy shows advantages in terms of hospital stay and cost compared to conventional VATS bullectomy. In fact, meta-analyses of lung cancer anatomical resection showed similar results for both nonintubated and conventional VATS bullectomy $(11,12)$. Based on the results of this study, non-intubated VATS bullectomy is a stable and advantageous procedure that can replace conventional VATS bullectomy.

When initially planning non-intubated VATS bullectomy, two decisions are required. The first concern is the choice of pain control for use during surgery. In general, epidural anesthesia and local anesthesia are available for non-intubated VATS bullectomy. Although epidural anesthesia can block most thoracic nerves and control chest wall and thoracic pain for a long time, it requires anesthetic preparation and an anesthesia recovery time similar to that for general anesthesia. Aside from the physiological advantage of non-intubated VATS, it is difficult to achieve an economic advantage over conventional VATS. On the other hand, local anesthesia using lidocaine does not have the same time requirements with respect to preparation for use in anesthesia. Therefore, quick surgery is possible and the time from admission to surgery can be minimized (13). The second concern is the choice of sedation method, which is the most important factor to control unexpected behavior of the patient during surgery and ensure a stable operation. At the planning stage, we considered the use of midazolam alone as a sedative. Midazolam for anesthesia has already been demonstrated to be safe and useful in drug-induced sleep endoscopy, being an anesthetic method that can safely bring the patient to a calm state without inhibiting the respiratory center in bullectomy. However, while midazolam has a sedative effect, it does not have an analgesic effect. In particular, when coughing is induced by vagal nerve stimulation due to excessive lung manipulation during surgery, the patient may wake from the calm state and complain of anxiety symptoms accompanied by 
dyspnea. Midazolam was deemed insufficient to induce sedation alone, so intravenous fentanyl was added for deeper sedation (14). The addition of fentanyl could solve the problem of coughing due to vagal nerve stimulation, which is the most difficult problem associated with non-intubated VATS. However, the depth of sedation due to the addition of fentanyl tends to decrease respiration (15). In deep sedation, oxygen supply through the nasal cannula or mask may sometimes become unstable. Therefore, it is necessary to maintain oxygen saturation through more efficient oxygen supply. Recently, high-flow nasal cannulas have been used as substitutes for mechanical ventilators in the treatment of dyspnea. Therefore, the application of a high-flow nasal cannula was considered to be a suitable alternative.

Nasal cannulas or masks cannot provide an equal supply of oxygen to the alveoli when the patient's breathing becomes unstable, or in cases where the collapse of the surgical lung is severe; that is, if the patient's condition is unstable during unexpected surgical situations, stable oxygen supply can be difficult so intubation may be necessary. The high-flow nasal cannula is an open gas delivery system. The high flow overcomes the patient's breath and generates positive pressure through the nasal cavity. Although the pressure is relatively low compared to a closed system, it is appropriate to inflate collapsed alveoli and maximize lung capacity. The difference between the inspiratory volume of the patient and the amount of gas flow delivered is small, and $\mathrm{FiO}_{2}$ is applied relatively constantly. The advantage of this high flow $\mathrm{O}_{2}$ therapy is that it can generate high positive oxygen concentration by generating positive pressure in alveoli via spontaneous respiration during nonintubated VATS (16). The application of a high-flow nasal cannula maintained stable oxygen saturation throughout the operation. None of the patients had oxygen saturation $<95 \%$ during the operation, and there were no cases in which tachycardia, tachypnea, or hypotension occurred. Coughing by vagal nerve stimulation during surgery was also reduced by the depth of sedation, and stable surgical procedures without vagal nerve block were possible in all cases.

The results of the present study indicated that nonintubated VATS bullectomy using a high-flow nasal cannula is as safe and stable as conventional VATS bullectomy. As $70 \%$ of the surgeries were performed by a surgeon with less than 2 years of clinical experience, the learning time for the transition from general anesthesia to non-intubated VATS would be short for most thoracic surgeons. The results of non-intubated VATS bullectomy were similar to those of conventional VATS bullectomy with regard to postoperative bleeding, duration of chest tube insertion, incidence of complications, recurrence rate, and number of days hospitalized. Therefore, non-intubated VATS bullectomy can replace conventional VATS bullectomy. In addition, non-intubated VATS increase the efficiency of OR use. Although there may be differences among hospitals, the number of ORs is generally insufficient to fully meet the requirements of surgeons, and so the effective use of these limited resources is required. The OR use time is influenced by the time from entering the OR to skin incision, operation time, and the time to awaken from anesthesia. Non-intubated VATS with local anesthesia can reduce the time from entering the OR to skin incision, and the time to awaken from anesthesia (17). Our results indicated that we could reduce the time spent in the OR. Therefore, applying the non-intubated VATS technique using local anesthesia can be an effective means of improving the efficiency of OR use.

Non-intubated VATS through local anesthesia was also beneficial in terms of cost of treatment and OR use efficiency. As there is no need for general anesthesia, the economic benefit of this method is high. First, there is a decrease in the length of hospital stay. There are economic benefits of both decreasing the length of time from admission to the beginning of surgery and decreasing the period from surgery to discharge. When choosing general anesthesia, the timing of the surgery is influenced by the OR schedule and the anesthesia schedule. However, in the case of non-intubated VATS through local anesthesia, this process is not applicable and the time from admission to surgery can thus be minimized. In addition, as use of local anesthesia reduces the time needed to recover, it takes less time to return to activities of daily life, such as ambulation. Compared to the general anesthesia group, in which the average time to ambulation was 1 day, the non-intubated VATS group with local anesthesia showed ambulation on the day of operation. In addition, the operation cost was reduced by avoiding use of general anesthesia $(2,11,12,18,19)$.

We thought the limitations of this study are relatively small number of cases in each group, retrospective study, and mean follow up duration was shorter in the local anesthesia group. The shorter follow up duration of the local anesthesia group can be related with lower recurrence rate after the operation than the general anesthesia group.

\section{Conclusions}

In conclusion, non-intubated VATS bullectomy through 
local anesthesia not only confers pathophysiological advantages, but is also advantageous with regard to cost and efficiency of OR use, owing to the omission of general anesthesia. There were no differences in clinical short-term outcomes or surgical results between non-intubated and conventional VATS bullectomy, although there have been no reports of long-term outcomes of non-intubated VATS bullectomy with local anesthesia. Non-intubated VATS with local anesthesia seems to be a promising alternative to VATS bullectomy using general anesthesia.

\section{Acknowledgments}

None.

\section{Footnote}

Conflicts of Interest: The authors have no conflicts of interest to declare.

Ethical Statement: The authors are accountable for all aspects of the work in ensuring that questions related to the accuracy or integrity of any part of the work are appropriately investigated and resolved. This study was approved by the Institutional Review Board of Pusan National University Yangsan Hospital (IRB-No. 05-2019012) and written informed consent was obtained from all patients.

\section{References}

1. Pompeo E. State of the art and perspectives in nonintubated thoracic surgery. Ann Transl Med 2014;2:106.

2. Gonzalez-Rivas D, Bonome C, Fieira E, et al. Nonintubated video-assisted thoracoscopic lung resections: the future of thoracic surgery? Eur J Cardiothorac Surg 2016;49:721-31.

3. Liu YJ, Hung MH, Hsu HH, et al. Effects on respiration of nonintubated anesthesia in thoracoscopic surgery under spontaneous ventilation. Ann Transl Med 2015;3:107.

4. Navarro-Martinez J, Galvez C, Rivera-Cogollos MJ, et al. Intraoperative crisis resource management during a nonintubated video-assisted thoracoscopic surgery. Ann Transl Med 2015;3:111.

5. Rocco G. Non-intubated uniportal lung surgerydagger. Eur J Cardiothorac Surg 2016;49 Suppl 1:i3-5.

6. Furak J, Szabo Z, Horvath T, et al. Non-intubated, uniportal, video assisted thoracic surgery (VATS) lobectomy, as a new procedure in our department. Magy
Seb 2017;70:113-7.

7. Irons JF, Martinez G. Anaesthetic considerations for nonintubated thoracic surgery. J Vis Surg 2016;2:61.

8. Pompeo E. Awake thoracic surgery--is it worth the trouble? Semin Thorac Cardiovasc Surg 2012;24:106-14.

9. Rocco G, La Rocca A, Martucci N, et al. Awake singleaccess (uniportal) video-assisted thoracoscopic surgery for spontaneous pneumothorax. J Thorac Cardiovasc Surg 2011;142:944-5.

10. Pompeo E, Tacconi F, Mineo D, et al. The role of awake video-assisted thoracoscopic surgery in spontaneous pneumothorax. J Thorac Cardiovasc Surg 2007;133:786-90.

11. Tacconi F, Pompeo E. Non-intubated video-assisted thoracic surgery: where does evidence stand? J Thorac Dis 2016;8:S364-75.

12. Deng HY, Zhu ZJ, Wang YC, et al. Non-intubated video-assisted thoracoscopic surgery under loco-regional anaesthesia for thoracic surgery: a meta-analysis. Interact Cardiovasc Thorac Surg 2016;23:31-40.

13. Guo Z, Yin W, Wang W, et al. Spontaneous ventilation anaesthesia: total intravenous anaesthesia with local anaesthesia or thoracic epidural anaesthesia for thoracoscopic bullectomy. Eur J Cardiothorac Surg 2016;50:927-32.

14. Zhao ZR, Lau RWH, Ng CSH. Anaesthesiology for uniportal VATS: double lumen, single lumen and tubeless. J Vis Surg 2017;3:108.

15. Okuda K, Nakanishi R. The non-intubated anesthesia for airway surgery. J Thorac Dis 2016;8:3414-9.

16. Baudin F, Buisson A, Vanel B, et al. Nasal high flow in management of children with status asthmaticus: a retrospective observational study. Ann Intensive Care 2017;7:55.

17. Hwang J, Shin JS, Son JH, et al. Non-intubated thoracoscopic bullectomy under sedation is safe and comfortable in the perioperative period. J Thorac Dis 2018;10:1703-10.

18. Umari M, Falini S, Segat M, et al. Anesthesia and fasttrack in video-assisted thoracic surgery (VATS): from evidence to practice. J Thorac Dis 2018;10:S542-s54.

19. Mineo TC, Tamburrini A, Perroni G, et al. 1000 cases of tubeless video-assisted thoracic surgery at the Rome Tor Vergata University. Future Oncol 2016;12:13-8.

Cite this article as: Jung J, Kim DH, Son J, Lee SK, Son BS. Comparative study between local anesthesia and general anesthesia in the treatment of primary spontaneous pneumothorax. Ann Transl Med 2019;7(20):553. doi: 10.21037/ atm.2019.09.89 